RAFAEL BENJAMIN ARAÚJO DIAS

\title{
ANÁLISE DO TRANSCRIPTOMA (RNAseq) DAS CÉLULAS FOLICULARES TIREOIDIANAS REVELA UMA DIVERSIDADE DE AÇÕES AUTÓCRINAS DO T3
}




\section{RESUMO}

DIAS, R.B.A Análise do transcriptoma (RNAseq) das células foliculares tireoidianas revela uma diversidade de ações autócrinas do T3. 2018. f.89. Tese (Doutorado em Fisiologia Humana) - Instituto de Ciências Biomédicas, Universidade de São Paulo, 2018.

Os hormônios tireoidianos (HTs) desempenham um papel importante em diversos processos, tais como o crescimento, desenvolvimento e metabolismo dos tecidos em geral. Embora estudos tenham demonstrado que os HTs agem diretamente nas células foliculares da tireoide reduzindo sua resposta ao TSH, pouco se sabe, a nível molecular, sobre essa e outras ações dos HTs na própria glândula tireoide. Nesse sentido, o objetivo do presente trabalho foi avaliar alterações no perfil de expressão gênica nas células foliculares tireoidianas (células PCCl3) em reposta ao tratamento com triiodotironina (T3). Após atingir a confluência desejada, as células PCCl3 foram mantidas em meio depletado de HTs (grupo hipotireoideo - Hipo) por 24h. Após esse período, parte das células foi tratada com $10^{-7} \mathrm{M}$ de T3 (grupo T3) por $24 \mathrm{~h}$. As células foram, então, lisadas para extração de RNA total para análise do transcriptoma, por RNAseq. Foi obtido como resultado, uma lista de genes diferencialmente expressos da qual foram selecionados cinco genes para validação in vitro (novo lote de células $\mathrm{PCCl} 3$ submetidas às mesmas condições descritas acima) e in vivo [ratos Wistar (250g), tratados por 4 semanas com

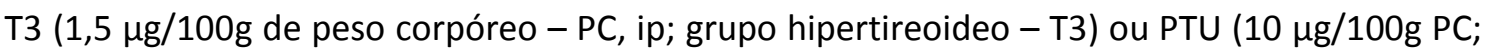
ip, grupo hipo): o S/c16a1, que codifica o MCT 8, responsável pelo transporte de T3 através da membrana, o Snrpd1, 9-March, Pfdn1 e Fam103a1, que codificam proteínas envolvidas no controle pós-transcricional e pós-traducional da expressão gênica. O tratamento com T3 estimulou a expressão dos genes Snrpd1, Pfdn1 e Fam103a1, enquanto reduziu a expressão de 9-March e S/c16a1. Juntos esses resultados demonstram a existência de um efeito autócrino exercido pelo T3 sobre o controle do seu próprio turnover proteico.

Palavras-chave: Hormônios tireoidianos. Sequenciamento de Nova Geração. Expressão Gênica. Células tireoidianas. Ratos Wistar. PTU. T3. 


\section{INTRODUÇÃo}

Ao longo dos anos, o conceito de homeostase foi sofrendo evoluções e modificações com o avanço da ciência e das novas tecnologias desenvolvidas para o estudo da fisiologia dos seres vivos. A ideia de que uma constância no ambiente interno era necessária para o desenvolvimento e a sobrevivência das diferentes formas de vida foi dando lugar à ideia de homeostase como um equilíbrio dinâmico de variáveis fisiológicas que flutuavam de maneira dependente ou independente do tempo, possibilitando assim a integração de diferentes processos que, juntos, garantiriam o sucesso da sobrevivência (1).

Nesse contexto, o sistema endócrino emerge como uma importante ferramenta capaz de integrar o funcionamento dos diferentes sistemas, por meio da síntese e liberação de uma ampla gama de hormônios que desempenharão suas funções nos mais variados tecidos, promovendo assim, o funcionamento homeostático do organismo, como um todo.

Dentre essa grande variedade de moléculas sinalizadoras encontram-se os hormônios tireoidianos, cuja síntese e secreção ocorrem nos folículos tireoidianos, constituídos basicamente pelas células foliculares tireoidianas e 0 coloide intrafolicular.

As células foliculares da tireoide sofrem um complexo programa de diferenciação que as torna as únicas células capazes de produzir os hormônios tireoidianos (2). Esses hormônios derivam da degradação da tireoglobulina (Tg), que é iodada em resíduos de tirosina pela ação da enzima tireoperoxidase (TPO) encontrada exclusivamente em células tiroidianas. A Tg iodada é então estocada no coloide do lúmen do folículo tiroideano e, sob a ação do hormônio tireotrófico (TSH) é endocitada pelo tirócito e hidrolisada, gerando T3 e T4 como produtos finais, os quais são posteriormente secretados na corrente sanguínea (2). Tg, TPO e receptor de TSH (TSHR) são os marcadores de diferenciação das células foliculares da tireoide (3).

A glândula tireoide, a hipófise e o hipotálamo constituem o eixo Hipotálamo-Hipófise-Tireoide (HHT), responsável pelo fino controle de síntese, secreção e regulação das concentrações de hormônios tireoidianos (HT) circulantes. $O$ processo que culminará com a liberação de T3/T4 na circulação sanguínea se inicia no núcleo paraventricular do hipotálamo, onde ocorre a 
síntese e secreção de TRH (Thyrotropin-Releasing Hormone), que age sobre os tireotrofos localizados na porção anterior da hipófise, estimulando a síntese e secreção de TSH, o principal hormônio regulador da função tireoidiana (4).

Na tireoide, o TSH interage com o TSHR deflagrando vias de sinalização intracelulares, que incluem a ativação da adenilil ciclase bem como da fosfolipase $C$, responsáveis pela geração de respostas que influenciam diversos eventos fisiológicos no controle do metabolismo da glândula bem como da expressão e atividade de importantes proteínas envolvidas com a via de síntese dos HT, como co-transportador $\mathrm{Na}^{+} / \mathrm{I}^{-}$(NIS), TPO, Tg e Dual oxidase (DUOX) $(5,6)$. O TSH promove também aumento do número e atividade das microvilosidades que se encontram na porção apical da célula folicular tireoideana, sendo fundamental para a endocitose, lise do coloide e, consequentemente, liberação dos HTs da Tg e secreção deles através do MCT 8.

A captação de iodeto da circulação sanguínea ocorre por meio da NIS, expressa na membrana basolateral das células foliculares tireoidianas e é resultado de um transporte simultâneo de dois íons $\mathrm{Na}^{+}$e um íon I' que se deslocam na mesma direção, possibilitando a entrada do iodeto no tireocito. A expressão e atividade do NIS são reguladas positivamente pelo TSH e negativamente pelo excesso de iodo; este último evento está relacionado ao fenômeno conhecido como Efeito Wolff-Chaickoff, que por sua vez se caracteriza por um bloqueio na organificação de $\mathrm{I}^{-}$concomitantemente com a interrupção da síntese e secreção de $\mathrm{HT}(7,8,9,10,11,12,13,14)$.

Uma vez no interior dos tireócitos o iodeto atravessa a membrana apical em direção ao coloide, onde ocorrerá o processo de organificação. Esse transporte é mediado pela anoctamina 1 (ANO1) em condições fisiológicas (15, 16) e, em condições de excesso de iodo, principalmente pela Pendrina (17).

No coloide, o iodeto é oxidado e covalentemente ligado em resíduos de tirosina da molécula de $\mathrm{Tg}$, processo catalizado pela enzima TPO, que utiliza $\mathrm{H}_{2} \mathrm{O}_{2}$ como agente oxidante do $\mathrm{I}^{-}$. A capacidade da glândula tireoide de produzir peróxido de hidrogênio se dá em função da expressão das enzimas DUOX (1 e 2), uma família de NADPH oxidases (18, 19, 20, 21, 22, 23).

$O$ processo de iodação da $\mathrm{Tg}$ gera principalmente monoiodotirosinas (MIT), diiodotirosinas (DIT), que se acoplam formando T3 (MIT + DIT) e T4 (DIT 
+ DIT). A Tg é então endocitada pelas microvilosidades da membrana apical das células foliculares e hidrolisada por proteases lisossomais que promovem a liberação das MITs, DITs, T3 e T4 da Tg para o citoplasma de onde, através de transportadores específicos, principalmente o MCT 8, o T3 e T4 atingem a corrente sanguínea.

Oitenta por cento dos HT são liberados na forma de T4 e $20 \%$ na forma de T3. Na circulação estes interagem com proteínas como a TBG (thyroxin binding globulin), TBPA (thyroid binding prealbumin) ou TTR (transthyretin) e albumina, sendo apenas $0,03 \%$ dos HT encontrados na forma livre. Essas proteínas transportadoras têm, na verdade, função de tamponamento ligandose aos HTs e aumentando, assim, a sua meia vida na circulação sanguínea. $(24,25,26)$.

Nos tecidos periféricos grande parte do T4 sofre ação de desiodases, que removem o iodo da posição 5' do seu anel externo gerando T3. Existem três isoformas de desiodases, as quais são expressas de maneira tecidoespecífica. A desiodase tipo I (D1) é altamente expressa em tecidos como fígado e rins e responsável, em sua maioria, pela conversão de T4 a T3 e geração de T3 para o plasma, principalmente em situações de aumento da concentração do T3 plasmático. Já a desiodase tipo II (D2) é encontrada principalmente no cérebro, hipófise e tecido adiposo marrom, sendo responsável pela conversão intracelular de T4 a T3, gerando T3 principalmente para consumo interno. Contudo, sabe-se que ela também é expressa em vários outros tecidos e, mais recentemente, tem sido atribuído à D2 o papel de igualmente gerar T3 para a periferia na condição de eutiroidismo. A desiodase tipo III (D3) é expressa na placenta, cérebro, fígado e pele e gera rT3, a partir de T4, e T2 a partir de T3, sendo considerada uma enzima inativadora dos HT (26).

Friesema e colaboradores (2003) foram os primeiros a demonstrar, através de injeção de cRNA em oócitos de Xenopus laevis, que alguns membros da família de transportadores de monocarboxilatos (MCTs: MonoCarboxylate Transporters) funcionavam como transportadores específicos de HT representando a principal porta de entrada de T3 nas células. A família de transportadores MCT é composta, originalmente, de 14 membros (MCT1- 
14) e transporta, principalmente, piruvato e lactato, sendo, portanto, de extrema importância no controle do metabolismo celular (27).

Uma vez no interior das células os HT interagem com seus receptores específicos localizados no núcleo, processo que é protagonizado pelo T3. Os receptores dos HT estão associados aos seus elementos responsivos (THRE) na molécula de DNA, mesmo na ausência de seu ligante. Outros receptores da superfamília dos receptores nucleares, como RXR (Receptor de retinóide X), se associam aos TRs formando heterodímeros. Todo esse complexo interage, por sua vez, com proteínas conhecidas como co-repressoras, que atuam inibindo a maquinaria de transcrição basal. A ligação do HT gera alterações conformacionais que possibilitam a dissociação das proteínas co-repressoras e a associação de proteínas co-ativadoras, que medeiam a transcrição dos genes alvo específicos em cada tecido. O mecanismo de repressão gênica induzida pelo T3 ainda não está totalmente definido, mas parece envolver o recrutamento de proteínas co-repressoras $(26,28,29,30)$. Tais efeitos são conhecidos como clássicos, genômicos ou nucleares. Contudo, um número crescente de estudos tem apontado para uma série de efeitos desencadeados pelo T3 que não são primariamente decorrentes de alterações na expressão gênica. Esses efeitos são chamados não clássicos ou não-genômicos. Caracteristicamente, as respostas a esses estímulos são rápidas, ocorrendo de segundos a minutos, e utilizam vias de sinalização deflagradas por receptores localizados na membrana plasmática ou no citossol.

Viu-se que os HT interagiam com uma família de glicoproteínas conhecidas como integrinas, especificamente com a isoforma av $\beta 3$. Caracteristicamente, são proteínas transmembranicas que apresentam um domínio extracelular capaz de interagir com elementos da matriz extracelular que apresentam o domínio RGD, formado pelos aminoácidos Arg-Gly-Asp, e um domínio intracelular ligado ao citoesqueleto. Esse domínio extracelular também funciona como sítio de reconhecimento de HT. Estudos subsequentes identificaram a presença de dois sítios nos quais os HT se ligavam na integrina, o S1 e o S2. O sítio S1 é reconhecido somente pelo T3 em concentrações fisiológicas conduzindo a ativação de Src e posteriormente de PI3K. Já o sítio S2 reconhece tanto T3 quanto T4 e leva a ativação da via $\operatorname{ERK1/2~(31,~32,~33,~}$ $34,35,36,37)$. As ações não genômicas desencadeadas no citossol decorrem 
da interação do HT com seu receptor (TRa ou $\beta$ ) acoplado a PI3K, por exemplo, mecanismo pelo qual o HT ativa essa enzima gerando efeitos como aumento de mTOR e da síntese proteica (38).

Atuando de maneira genômica ou não genômica é indiscutível que os hormônios tireoidianos asseguram o controle de uma série de processos fisiológicos que, em última instância, garantem o funcionamento homeostático do organismo.

Desde o período de desenvolvimento embrionário, se conhece o papel imprescindível que tais hormônios desempenham, especialmente na formação e maturação do Sistema Nervoso central (SNC), processo resultante da expressão de diferentes genes como os que codificam a proteína TAU, relacionada às projeções axonais dos neurônios, a MAP2, envolvida com a formação dos dendritos, além de relina, que é essencial para a correta citoarquitetura das estruturas cerebrais e BDNF, que exerce um papel essencial no desenvolvimento do Sistema Nervoso (39). Portanto, a deficiência de hormônios tireoidianos nesta fase de desenvolvimento, prejudica 0 crescimento neuronal comprometendo diversas funções do Sistema Nervoso Central, levando a um quadro de um acentuado grau de retardo mental conhecido como cretinismo (40, 41, 42, 43, 44, 45, 46, 47).

As ações dos hormônios tireoidianos não se restringem apenas à fase de desenvolvimento embrionário, na fase adulta eles também exercem uma série de efeitos modulatórios responsáveis por controlar eventos metabólicos de diversas naturezas.

A literatura reporta uma importante participação dos hormônios no controle do metabolismo dos carbohidratos através de diversos mecanismos. Indivíduos com hipertireoidismo apresentam intolerância à glicose, o mesmo ocorrendo nos estados de hipotireoidismo quadros de hiperglicemia, e um dos fatores que explicam essa relação é que existe, nesses indivíduos, uma redução da meia-vida da insulina concomitantemente com aumento da liberação de pró-insulina, devido a um incorreto processamento deste hormônio. Ao mesmo tempo em que essas alterações ocorrem nas células $\beta$ pancreáticas, no fígado, o HT aumenta a produção hepática de glicose e a expressão de GLUT2 na membrana plasmática dos hepatócitos, o qual é responsável por transportar glicose do fígado para a circulação. Esse $O$ 
excesso de HT gera também um aumento da lipólise, resultando em aumento de ácidos graxos livres (FFA-free fat acid) e glicerol na circulação, sendo que este último é um precursor da gliconeogênese hepática $(48,49,50,51,52)$. Quanto ao metabolismo das proteínas o T3 aumenta o turnover das mesmas, sendo que no hipertireoidismo o catabolismo protéico predomina.

No coração, o T3 também exerce importantes ações fisiológicas aumentando a atividade e expressão de uma série proteínas envolvidas com a contração ( $\mathrm{aMHC})$ e relaxamento $\left(\mathrm{SERCa^{+2 } )}\right.$ cardíacos. Nesse sentido, indivíduos hipotireoideos apresentam uma diminuição na expressão e atividade de SERCa ${ }^{+2}$, o que consequentemente acarreta na diminuição do intervalo de relaxamento diastólico, enquanto indivíduos hipertireoideos apresentam frequentemente hipertrofia cardíaca $(53,54)$.

Até o presente momento, a maioria dos estudos que visam entender as ações dos HT tem focado nos efeitos do hormônio sobre os mais diferentes tecidos, já que há evidências de que todos eles apresentam receptores de HT. No entanto, a glândula tireoide também expressa receptores de HT e poucos são os estudos que intentaram elucidar um possível papel deles na regulação da sua própria função. Pelo nosso conhecimento, há apenas um trabalho em que se mostra uma ação direta dos HT sobre as células foliculares da tireoide inibindo sua resposta ao TSH. Contudo quase nada se sabe, a nível molecular, sobre seu papel nos tireócitos (55), sendo esse um desafio que buscamos enfrentar com esse estudo.

Por mecanismos genômicos e/ou não-genômicos os hormônios tireoidianos exercem sua função através do controle da expressão de genes, ou da atividade de diferentes enzimas, que, por sua vez, levam à alterações na maquinaria de funcionamento celular, gerando respostas frente a determinados estímulos. Com a conclusão do Projeto Genoma em 2003, e o acúmulo exponencial de sequências gênicas e genomas depositados em bancos de dados públicos, a demanda por metodologias que permitam a identificação funcional, confirmação de homologia, elucidação de padrões de expressão, dentre outras funções tem crescido consideravelmente. Atualmente diversas metodologias tem surgido no intuito de se avaliar o perfil de expressão gênica de um determinado tecido ou célula frente a um determinado tratamento ou desafio. Dentre essas metodologias o Sequenciamento de Nova Geração - 
NGS vem ganhado muito espaço nos laboratórios e centros de pesquisa pois é capaz de gerar milhões de "short reads" por ensaio, o que torna essa metodologia útil para as mais variadas aplicações biológicas $(56,57,58,59)$.

Os primeiros trabalhos utilizando sequenciamento de DNA como ferramenta de estudo de expressão gênica apareceram em 1977 em trabalhos publicados por Fred Sanger e Alan R. Coulson. Desde então, as tecnologias de sequenciamento tem avançado de maneira expressiva nas últimas décadas.

O sequenciamento de Nova Geração (New Generation Sequencing NGS) tem como fundamento o sequenciamento de DNA em plataformas capazes de gerar milhares de informações sobre milhões de pares de bases em uma única etapa. As tecnologias de sequenciamento via NGS fornecem informações adicionais quando comparados com outros métodos de sequenciamento (Sanger), como estimação de abundância de expressão, detecção de múltiplos eventos de splicing alternativo, edição de sequencias de DNA e RNA, identificação de novos transcritos, dentre outros. O sequenciamento direto de RNA (RNA-seq) ainda não é possível com as plataformas comerciais de sequenciamento já existentes, contudo, é possível sequenciá-lo, de maneira indireta, pela transcrição reversa em cDNA. Assim, o RNA-seq é capaz de determinar, de maneira absoluta, a quantidade de moléculas de mRNA em uma condição específica e assim comparar resultados de experimentos independentes. Há várias plataformas de sequenciamento disponíveis, as mais utilizadas são $454 \mathrm{FLX}$ da Roche $\AA$, a Solexa da Illumina $\AA_{\text {, }}$ Heliscope True Single Molecule Sequencing (tSMS), da Helicos ${ }^{\circledR}$ e também, a SOLiD System, da Applied, plataforma que foi utilizada neste trabalho $(60,61$, $62,63,64,65)$.

De modo geral o SNG obedece a uma sequencia de passos que se iniciam com a extração do RNA total de amostras de células ou tecidos. Posteriormente o RNA total passa por um enriquecimento, no qual moléculas de RNAr, RNAt e outros pequenos RNAs são removidos das amostras e os fragmentos restantes são então quebrados em pequenos fragmentos (200-300 pares de base) denominados reads, que, em seguida são submetidos a reação de transcrição reversa para a construção das bibliotecas de cDNA. As amostras são então sequenciadas, e os dados analisados por bioinformática. 
Um dos passos importantes no processo de sequenciamento é o mapeamento dos reads; em inglês cunhou-se o termo mapping para designar a tarefa de se encontrar uma única localização para um determinado read no genoma de referência de uma determinada espécie. Entretanto, pequenos reads podem, muitas vezes, alinhar em diferentes localizações no mesmo genoma conduzindo a erros de sequenciamento que precisam ser resolvidos. Existem algumas ferramentas computacionais que auxiliam na solução desse problema. Estes softwares (SplitSeek, Tophat, SOAPals) utilizam uma estratégia de correspondência heurística de primeira passagem que rapidamente encontra uma lista de possíveis locais de alinhamento para um mesmo read, seguida por uma avaliação completa de todos os possíveis alinhamentos. Por ultimo, uma análise probabilística define qual o alinhamento correto, em meio a todas aquelas possibilidades, para este determinado read $(66,67)$.

Finalizado o mapeamento dos reads, a etapa imediatamente posterior é encontrar um significado biológico para eles (éxons, transcritos ou genes) através de uma abordagem que leva em conta o número de reads que se sobrepõem aos éxons de um determinado gene. Contudo, uma porção significativa desses reads mapeia regiões genômicas fora de éxons conhecidos, isso conduz à possibilidade de se encontrar novos transcritos, ou mesmo novos genes expressos.

Para a etapa de análise de genes diferencialmente expressos (GDE) que consiste em encontrar os genes que modificaram seus níveis de expressão nas diferentes condições experimentais, é necessária inicialmente uma normalização dos dados, a qual tem como objetivo tornar os valores de expressão gênica comparáveis, uma vez que as etapas de sequenciamento geram uma variação muito grande no número de reads produzidos em cada rodada de sequenciamento. Assim os procedimentos de normalização tentam contabilizar essas diferenças, possibilitando maior acuidade nas comparações entre grupos de amostras. Existem diversos pacotes de normalização disponíveis nos diferentes pacotes estatísticos para análises, dentre eles o DESeq, DEGSeq, edgeR e baySeq $(68,69)$.

Nas últimas décadas, várias metodologias têm sido propostas para a análise de genes diferencialmente expressos em um estudo de RNA-seq. Os 
dados de RNA-seq obedecem a um padrão de distribuição discreta para cada gene. Duas distribuições discretas de probabilidades têm sido utilizadas para modelar dados de sequenciamento por RNA-seq: Distribuição de Poisson e Binomial Negativa. A grande desvantagem na utilização da distribuição de Poisson é que ela não leva em conta a variabilidade biológica entre as amostras, o que acarreta em altas taxas de falsos positivos em razão de desconsiderar ou subestimar o erro experimental; logo não é de se surpreender que a adição de replicatas biológicas seja de fundamental importância quando se planeja um experimento de sequenciamento. Em função disso a Binomial negativa, que leva em consideração essa variabilidade experimental, é a ferramenta mais utilizada atualmente para a análise de dados de sequenciamento. Dentre os pacotes disponíveis estão: baySeq, DESeq, DEGSeq e edgeR $(70,71,72)$.

Baseados nesses antecedentes, buscamos com esse estudo avaliar a expressão diferencial de genes na tireoide em resposta ao T3, por meio de RNAseq, com a finalidade de identificarmos as ações autócrinas deste hormônio e sua possível relevância para a função tireoidiana. 


\section{CONCLUSÃO}

Através de uma abordagem de avaliação de expressão gênica em larga escala (sequenciamento de nova geração), o presente trabalho ilustra, pela primeira vez, a nível molecular, a existência de uma ação autócrina exercida pelo T3 regulando a expressão de uma série de genes envolvidos em diversos eventos fisiológicos da glândula. Dentre os genes selecionados para a validação do experimento de transcriptoma verificou-se que muitos dos genes regulados pelo T3 estão envolvidos em eventos pós-transcricionais e póstraducionais, o que indica uma crucial participação do T3 no controle da sua massa, vascularização e função, em paralelo aos efeitos do próprio TSH. Apesar de muitas das validações feitas neste trabalho, terem focado apenas no estudo de expressão na própria tireoide, os referidos genes apresentam expressão ubíqua dentre os diversos tecidos, o que levanta discussões a respeito de uma possível influência do T3 regulando a ocorrência desses eventos pós-transcricionais e pós-traducionais não somente na tireoide, mas também, e outros tecidos, o que deve ser motivo de futuras investigações. 


\section{REFERENCIAS}

1- Recordati, G.; Bellini TG. A definition of internal constancy and homeostasis in the context of non-equilibrium thermodynamics. Exp Physiol.,v.89, p.27-38, 2004.

2- Chen, L.; Xu, B.; Liu, L.; Luo, Y.; Zhou, H.; Chen, W.; Shen, T.; Han, X.; Kontos, C.D.; Huang, S. Cadmium induction of reactive oxygen species activates the mTOR pathway, leading to neuronal cell death. Free Radic Biol Med., v.50, p.624-632, 2011.

3- Dumont, J.E.; Lamy, F.; Roger, P.; Maenhaut, C. Physiological and pathological regulation of thyroid cell proliferation and differentiation by thyrotropin and other factors. Physiol Rev., v.72, p.667-97, 1992.

4-Vassart, G.; Dumont, J.E. The thyrotropin receptor and the regulation of thyrocyte function and growth. Endocr Rev., v.13, p.596 - 611,1992.

5-Laugwitz, K.L.; Allgeier, A.; Offermanns, S.; Spicher, K.; Van Sande, J.; Dumont, J.E.; Schultz, G. The human thyrotropin receptor: a heptahelical receptor capable of stimulating members of all four G protein families. Proc Natl Acad Sci U S A. v.93, p.116-20, 1996.

6- Latif, R.; Morshed, S. A.; Zaidi, M.; Davies, T. F.TheThyroid-Stimulating Hormone Receptor: Impact of Thyroid-Stimulating Hormone and ThyroidStimulating Hormone Receptor Antibodies on Multimerization, Cleavage and Signaling. Endocrinol Metab Clin N Am., v.38, p.319-341, 2009.

7- Zimmermann, M.B.; Jooste, P.L.; Pandav, C.S.lodine-deficiency disorders. Lancet., v.372, p.1251-1262, 2008.

8- Nicola, J.P.; Basquin, C.; Portulano, C.; Reyna-Neyra, A.; Paroder, M.; Carrasco, $\mathrm{N}$. The $\mathrm{Na}+/ /-$ symporter mediates active iodide uptake in the intestine. Am J Physiol Cell Physiol., v.296, p.654-662, 2009.

9- Dai, G.; Levy, O.; Carrasco, N. Cloning and characterization of the thyroid iodide transporter. Nature., v.379, p.458-460, 1996.

10- Bizhanova, A.; Kopp, P. The sodium-iodide symporter NIS and pendrin in iodide homeostasis of the thyroid. Endocrino. v.150, p.1084-1090, 2009.

11- Uyttersprot, N.; Pelgrims, N.; Carrasco, N.; Gervy, C.; Maenhaut, C.; Dumont, J.E.; Miot, F.Moderate doses of iodide in vivo inhibit cell proliferation

\footnotetext{
*De acordo com: ASSOCIAÇÃO BRASILEIRA DE NORMAS TÉCNICAS. NBR 6023: Informação e documentação: referências: elaboração. Rio de Janeiro, 2002, 24p.
} 
and the expression of thyroperoxidase and $\mathrm{Na}+/ \mathrm{l}-$ symporter mRNAs in dog thyroid. Mol Cell Endocrinol., v.131, p.195-203, 1997.

12-Eng, P.H.; Cardona, G.R.; Fang, S.L.; Previti, M.; Alex, S.; Carrasco, N.; Chin, W.W.; Braverman, L.E. Escape from the acute Wolff-Chaikoff effect is associated with a decrease in thyroid sodium/iodide symporter messenger ribonucleic acid and protein. Endocrinology. v.140, p.3404- 3410, 1999.

13-Eng, P.H.; Cardona, G.R.; Previti, M.C.; Chin, W.W.; Braverman, L.E. Regulation of the sodium iodide symporter by iodide in FRTL-5 cells. Eur $\mathbf{J}$ Endocrinol., v.144, p.139-144, 2001.

14-Wolff, J.; Chaikoff, I.L. Plasma inorganic iodide as a homeostatic regulator of thyroid function. J Biol Chem., v.174, p.555-564, 1948.

15- Iosco, C.; Cosentino, C.; Sirna, L.; Romano, R.; Cursano, S.; Mongia, A.; Pompeo, G.; di Bernardo, J.; Ceccarelli, C.; Tallini, G.; Rhoden, K.J. Anoctamin 1 is apically expressed on thyroid follicular cells and contributes to ATP-and calcium-activated iodide efflux. Cell Physiol Biochem., v.34, p.966-80, 2014.

16- Twyffels, L.; Strickaert, A.; Virreira, M.; Massart, C.; Van Sande, J.; Wauquier, C.; Beauwens, R.; Dumont, J.E.; Galietta, L.J.; Boom, A.;, Kruys, V. Anoctamin-1/TMEM16A is the major apical iodide channel of the thyrocyte. Am J Physiol Cell Physiol., v.307, p.1102-12, 2014.

17- Calil-Silveira, J.; Serrano-Nascimento, C.; Kopp, P. A.; Nunes, M.T. lodide excess regulates its own efflux: a possible involvement of pendrin. Am J

Physiol Cell Physiol., v.310, p.576-582, 2016.

18- Corvilain, B.; Collyn, L.; Van Sande, J.; Dumont, J.E. Stimulation by iodide of $\mathrm{H} 2 \mathrm{O} 2$ generation in thyroid slices from several species. Am J Physiol Endocrinol Metab. v.278, p.692-699, 2000.

19-Panneels, V.; Van den Bergen, H.; Jacoby, C.; Braekman, J.C.; Van Sande, J.; Dumont, J.E.; Boeynaems, J.M. Inhibition of $\mathrm{H} 2 \mathrm{O} 2$ production by iodoaldehydes in cultured dog thyroid cells. Mol Cell Endocrinol., v.102, p.167-176, 1994.

20-Pisarev, M.A.; Gartner, R. Autoregulatory action of iodine. The thyroid., v. 9, p.85-90, 2000.

21-Panneels, V.; Juvenal, G.; Boeynaems, J.M.; Dumont, J.E.; Van Sande, J. lodide effects on the thyroid. Comprehensive handbook of iodine: nutritional, endocrine, and pathological aspects. Academic Press., p. 99305-99316, 2009.

22-Nunez ,J.; Pommier, J. Formation of thyroid hormones. Vitam Horm., v. 39, p.175-229, 1982. 
23-Corvilain, B.; Van Sande, J.; Laurent, E.; Dumont, J.E. The H2O2-generating system modulates protein iodination and the activity of the pentose phosphate pathway in dog thyroid. Endocrinology., v.128, p.779-785, 1991.

24- Vijlder, J.J.; Ris-stalpers, C.; Vulsma, T. Inborn errors of thyroid hormone biosynthesis. Exp Clin Endocrinol Diabetes., v.4, p.32-37, 1997.

25-Kopp, P. Pendred's syndrome and genetic defects in thyroid hormone synthesis. Rev Endocr Metabolic Disorders., v.1, p.109-121, 2000.

26- Yen, P. Physiological and Molecular Basis of Thyroid Hormone Action Physiological Reviews., v. 81, p. 234-260, 2001.

27- Friesema, E.C.; Ganguly, S.; Abdalla, A. Identification of monocarboxylate transporter 8 as a specific thyroid hormone transporter. The Journal of Biological Chemistry., v. 278, p.40128 40135, 2003.

28-Horlein, A.J.; Naar, A.M.; Heinzel, T.; Torchia, J.; Gloss, B.; Kurokawa, R.; Ryan, A.; Kamel, Y.; Soderstrom, M.; Glass, C.K.; Rosenfield, M.G. Ligandindependent repression by the thyroid hormone receptor mediated by a nuclear receptor co-repressor. Nature., v.377, p. 397-404, 1995.

29-Glass, C.K.; Rosenfeld, M.G. The coregulator exchange in transcriptional functions of nuclear receptors. Genes Dev., v.14, p.121-41, 2000.

30-Privalsky, M.L. The role of correpressor in transcriptional regulation by nuclear hormone receptors. Annu. Rev. Physiol., v.66, p.315-360, 2004.

31- Plow, E.F.; Haas, T.A.; Zhang, L.; Loftus, J.; Smith, J.W. Ligand binding to integrins. J Biol Chem., v. 275, p.21785-21788, 2000.

32- Calderwood, D.A.; Shattil, S.J.; Ginsberg, M.H. Integrins and actin filaments: reciprocal regulation of cell adhesion and signaling. J Biol Chem., v. 275, p.22607-22610, 2000.

33- Farwell, A.P.; Tranter, M.P.; Leonard, J.L.Thyroxine-dependent regulation of integrin-laminin interactions in astrocytes. Endocrinology., v.136, p.39093915, 1995.

34- Hood, J.D.; Frausto, R.; Kiosses, W.B.; Schwartz, M.A.; Cheresh, D.A. Differential $v$ integrin-mediated Ras-ERK signaling during two pathways of angiogenesis. J Cell Biol., v.162, p.933-943, 2003.

35- Pereira, J.J.; Meyer, T.; Docherty, S.E.; Reid, H.H.; Marshall, J.; Thompson, E.W.; Rossjohn, J.; Price, J.T. Bimolecular interaction of insulin-like growth 
factor (IGF) binding protein-2 with v3 negatively modulates IGF-I-mediated migration and tumor growth. Cancer Res., v.64, p.977-984, 2004.

36- Hoffman, S.J.; Vasko-Moser, J.; Miller, W.H.; Lark, M.W.; Gowen, M.; Stroup, G. Rapid inhibition of thyroxine-induced bone resorption in the rat by an orally active vitronectin receptor antagonist. J Pharmacol Exp Ther., v. 302, p.205-211, 2002.

37-Moeller, L.C.; Broecker-Preuss, M. Transcriptional regulation by nonclassical action of thyroid hormone. Thyroid Res., v.4, p.1-6, 2011.

38-Cao, X.; Moeller, L.C.; Dumitrescu, A.M.; Seo, H.; Refetoff, S. Thyroid hormone mediated changes in gene expression can be initiated by Cytosolic action of the thyroid hormone receptor beta through the Phosphatidylinositol 3-kinase pathway. Nucl Recept Signal. v.4, 2006.

39- Thomas, G.; Jacobs, K.B.; Kraft, P.; Yeager, M.; Wacholder, S.; Cox, D.G.; Hankinson, S.E.; Hutchinson, A.; Wang, Z.; Yu, K.; Chatterjee, N.; GarciaClosas, M.; Gonzalez-Bosquet, J.; Prokunina-Olsson, L.; Orr, N.; Willett, W.C.; Colditz, G.A.; Ziegler, R.G.; Berg, C.D.; Buys, S.S.; McCarty, C.A.; Feigelson, H.S.; Calle, E.E.; Thun, M.J.; Diver, R.; Prentice, R.; Jackson, R.; Kooperberg, C.; Chlebowski, R.; Lissowska, J.; Peplonska, B.; Brinton, L.A.; Sigurdson, A.; Doody, M.; Bhatti, P.; Alexander, B.H.; Buring, J.; Lee, I.M.; Vatten, L.J.; Hveem, K.; Kumle, M.; Hayes, R.B.; Tucker, M.; Gerhard, D.S.; Fraumeni, J.F. Jr.; Hoover, R.N.; Chanock, S.J.; Hunter, D.J. A multistage genome-wide association study in breast cancer identifies two new risk alleles at $1 \mathrm{p} 11.2$ and 14q24.1 (RAD51L1). Nat Genet.,v.41, p.579-84, 2009.

40- Eayrs, J.T. Thyroid and developing brain: anatomical and behavioural effects. In: Hamburgh M, Barrington EJW, editors. Hormones and development., p.345-55, 1971.

41- Legrand J. Variations en fonction de l'âge de la response du cervelet à l'action morphogénétique de la thyroïde chez le rat. Arch Anat Microsc Morphol Exp., v.56, p.291-307, 1967.

42-Graves, C.; Hawkes, R. Maturation of the corpus callosum of the rat.I. Influence of thyroid hormones on the topography of callosal projections. I Comp Neurol. v.291, p.128-46, 1990.

43-Berbel, P.; Guadaño-Ferraz, A.; Martinez, M.; Quiles, J.A.; Balboa, R.; Innocenti, G.M. Organization of auditory callosal connections in hypothyroid adult rats. Eur J Neurosci., v.5, p.1465-78, 1993. 
44- Aniello, F.; Couchie, D.; Bridoux, A.M.; Gripois, D.; Nunez J. The splicing of juvenile and adult Tau mRNA variants is regulated by thyroid hormone. Proc Nati Acad Sci USA., v.88, p.4035-4039, 1991.

45-Mareck, A.; Fellous, A.; Francon, J.; Nunez, J. Changes in composition and activity of microtubule-associated proteins during brain development.

Nature.,v.284, p.353-5, 1980.

46-Lee, G.; Cowan, N.; Kirschner, M. The primary structure and heterogeneity of tau protein from mouse brain. Science., v.239, p.285-8, 1988.

47-Kosik, K.S.; Orecchio, L.D.; Bakalis, S.; Neve, R.L. Developmentally regulated expression of specific tau sequences. Neuron., v.2, p.1389-97, 1989.

48-Maxon, H.R.; Kreines, K. W.; Goldsmith, R. E.; Knowles. H. C. Long-term observations of glucose tolerance in thyrotoxic patients. Archives of InternalMedicine., v.135, p.1477-1480, 1975.

48- O’Meara, N.M.; Blackman, J. D.; Sturis, J.; Polonsky. K. S. Alterations in the kinetics of C-peptide and insulin secretion in hyperthyroidism. Journal of Clinical Endocrinology and Metabolism., v.76, p.79-84, 1993.

50-Dimitriadis,G.; Baker, B.; Marsh H. Effect of thyroid hormone excess on action, secretion, and metabolism of insulin in humans. The American journal of physiology., v.248, p. 593-601,1985.

51-Levin, R.J.; Smyth, D. H. The effect of the thyroid gland on intestinal absorption of hexoses. The Journal of physiology., v.169, p.755-769, 1963.

52- Matty, A.J.; Seshadri, B. Effect of thyroxine on the isolated rat intestine. Gut., v.6, p.200-202, 1965.

53- Vargas-Uricoecheaa, H.; Bonelo-Perdomob, A.; Hernán Sierra-Torresc, C. Effects of thyroid hormones on the heart. Clin Invest Arterioscl., v.26, p.296309. 2016.

54-Grais, I. M.; Sowers, J.R. Thyroid and the Heart. The American Journal of Medicine., v.127, p. 691-698, 2014.

55- Cortell, R.; Rawson, R.W. The effect of thyroxin on the response of the thyroid gland to thyrotropic hormone. Endocrinology., v.35, p.488-498, 1994.

56- Neves, C.E. Experimentos de microawways e teoria da resposta ao item. Universidade de São Paulo. 10.11606/D.45.2010.tde-24052010-140944. 2011.

57- Davies, K.E. The Application of DNA Recombinant Technology to the Analysis of the Human Genome and Genetic Disease. Hum Genet., v.58, p.351-357,1981. 
58-Kvam, V.M.; Liu, P.; Si, Y. A comparison of statistical methods for detecting differentially expressed genes from RNA-seqdata. Am J Bot., v.99, p.248-56, 2012.

59- Silva, D. C. G.; Carvalho, M. C. C. G. Sequenciamento de DNA de nova geração e suas aplicações na genômica de plantas. Ciencia Rural., v.40, p.735-744, 2010.

60- Marioni, J.C.; Mason, C.E.; Mane, S.M.; Stephens, M.; Gilad, Y. RNA-seq: An assessment of technical reproducibility and comparison with gene expression arrays. Genome research., v.18, p.1509-1517, 2008.

61-Morozova, O.; Hirst, M.; Marra, M.A. Applications of new sequencing technologies for transcriptome analysis. Annu Rev Genomics Hum Genet., v.10, p.135-51, 2009.

62-Wang, Z.; Gerstein, M.; Snyder, M. RNA-Seq: a revolutionary tool for transcriptomics. Nat Rev Genet., v.10, p.57-63, 2009.

63-Auer, P.L.; Doerge, R.W. Statistical design and analysis of RNA sequencing data. Genetics., v. 185, p. 405-16, 2010.

64- Carvalho, M. C. C. G.; Silva, D. C. G. Sequenciamento de DNA de nova geração e suas aplicações na genômica de plantas. Ciencia Rural., v.40, p.735-744, 2010.

65- Neves, C.E. Experimentos de microawways e teoria da resposta ao item. Universidade de São Paulo. 10.11606/D.45.2010.tde-24052010-140944. 2011.

66- Bao, H.; Guo, H.; Wang, J.; Zhou, R.; Lu, X.; Shi, S. MapView: visualization of short reads alignment on a desktop computer. Bioinformatics., v.25., p. 1554-5, 2009.

67- Oshlack, A.; Robinson, M.D.; Young, M.D. From RNA-seq reads to differential expression results. Genome Biol., v.11, p.220-230, 2010.

68- Bullard, J.H.; Purdom, E.; Hansen, K.D.; Dudoit, S. Evaluation of statistical methods for normalization and differential expression in mRNA-Seq experiments. BMC Bioinformatics., v.11, p.94-120, 2010.

69-Robinson, M.D.; McCarthy, D.J.; Smyth, G.K. edgeR: a Bioconductor package for differential expression analysis of digital gene expression data.

Bioinformatics., v.26, p.139-40, 2010. 
70- Kvam, V.M.; Liu, P.; Si, Y. A comparison of statistical methods for detecting differentially expressed genes from RNA-seq data. Am J Bot., v.99, p.248-256, 2012.

71-Langmead, B. Aligning short sequencing reads with Bowtie. Curr Protoc Bioinformatics., v.11, p.32-45, 2010.

72-Anders, S.; Huber, W. Differential expression analysis for sequence count data. Genome Biol., v.11, p.106-116, 2010.

73-Fusco, A.; Grieco, M.; Santoro, M.; Berlingieri, M.T.; Pilotti, S.; Pierotti, M.A.; Della Porta, G.; Vecchio G. A new oncogene in human thyroid papillary carcinomas and their lymph-nodal metastases. Nature., v.328, p.170-172, 1987.

74- Pan, Q.; Shai, O.; Lee, L.J.; Frey, B.J.; Blencowe, B.J. Deep surveying of alternative splicing complexity in the human transcriptome by high-throughput sequencing. Nat Genet., v. 40, p.1413-1415, 2008.

75-Sultan, M.; Schulz, M.H.; Richard, H.; Magen, A.; Klingenhoff. A.; Scherf, M.; Seifert, M.; Borodina, T.; Soldatov, A.; Parkhomchuk, D.; Schmidt, D.; O'Keeffe, S.; Haas, S.; Vingron, M.; Lehrach, H.; Yaspo, M.L. A global view of gene activity and alternative splicing by deep sequencing of the human transcriptome. Science., v.321, p.956-960, 2008.

76-Wagner, J.R.; Ge, B.; Pokholok, D.; Gunderson, K.L.; Pastinen, T.; Blanchette, M. Computational analysis of whole-genome differential allelic expression data in human. PLoS Comput Biol., 2010.

77-Wang, X.; Sun, Q.; McGrath, S.D.; Mardis, E.R.; Soloway, P.D.; Clark, A.G. Transcriptome-wide identification of novel imprinted genes in neonatal mouse brain. PLoS ONE., 2008.

78- Carvalho, M. C. C. G.; Silva, D. C. G. Sequenciamento de DNA de nova geração e suas aplicações na genômica de plantas. Ciencia Rural., v.40, p.735-744, 2010.

79- Farias, D.R.; Woyann, L.G.; Maia, L.C.; Oliveira, A.C. Análise comparativa de ferramentas de bioinformática para montagem de genomas com tecnologia de sequenciamento de nova geração. Vix Enpos, 2012.

80- Mckernan, K. Reagents, methods, and libraries for bead-based sequencing. US patent application 20080003571,2006. 
81- Durfee, T.; Nelson, R.; Baldwin, S.; Plunkett, G.; Burland, V.; Mau, B.; Petrosino, J.F.; Qin, X.; Muzny, D.M.; Ayele, M.; Gibbs, R.A.; Csörgo, B.;

Pósfai, G.; Weinstock, G.M.; Blattner, F.R. The complete genome sequence of Escherichia coli DH10B: insights into the biology of a laboratory workhorse. Journal of bacteriology., v.190, p.2597-2606, 2008.

82-Cloonan, N.; Forrest, A.R.; Kolle, G.; Gardiner, B.B.; Faulkner, G.J.; Brown, M.K.; Taylor, D.F.; Steptoe, A.L.; Wani, S.; Bethel, G.; Robertson, A.J.; Perkins, A.C.; Bruce, S.J.; Lee, C.C.; Ranade, S.S.; Peckham, H.E.; Manning, J.M.; McKernan, K.J.; Grimmond, S.M. Stem cell transcriptome profiling via massivescale mRNA sequencing. Nat Methods., v.7, p.613-9, 2008.

83-Passalacqua, K.D.; Varadarajan, A.; Ondov, B.D.; Okou, D.T.; Zwick, M.E.; Bergman, N.H. Structure and complexity of a bacterial transcriptome. $\mathbf{J}$ Bacteriol., v.191, p.3203-11, 2009.

84-Tang, F.; Barbacioru, C.; Wang, Y.; Nordman, E.; Lee, C.; Xu, N.; Wang, X.; Bodeau, J.; Tuch, B.B.; Siddiqui, A.; Lao, K.; Surani, M.A. mRNA-Seq wholetranscriptome analysis of a single cell. Nat Methods., v.6, p.377-82, 2009.

85-Santillo, A.; Burrone, L.; Falvo, S.; Senese, R.; Lanni, A.; Chieffi Baccari, G. Triiodothyronine induces lipid oxidation and mitochondrial biogenesis in rat Harderian gland. J Endocrinol., v.219, p.69-78,2013.

86-Eshak, M.G.; Hassan, W.A. Modulation of nitric oxide synthase and superoxide dismutase gene expression by altered thyroid levels in adult rat brain. Int J Pharm., v.4, p.10-23,2014.

87-Alexander, N. M. lodide peroxidase in rat thyroid and salivary glands and its inhibition by antithyroid compounds. J Biol Chem., v.234, p.1530,1959.

88- Slingerland, D. W.; Graham, D. E.; Josephs, R. K.; Yamazaki, E. The effect of propylthiouracil on the conversion of monoiodotyrosine to diiodotyrosine, Endocrinology., v.65, p.178, 1959.

89-Richards, J. B.; Ingbar, S.H. The effects of propylthiouracil and perch]orate on the biogenesis of thyroid hormone. Endocrinology., v.65, p.198, 1959.

90-lino, S.; Yamada, T.; Greer, M.A. Effect of graded doses of propylthiouracil on biosynthesis of thyroid hormones. Endocrinology., v.68, p.582, 1961.

91-Morris, D. R.; Hager, L.P. Mechanism of the inhibition of enzymatic halogenation by antithyroid agents. J Biol Chem., v.241, p.3582, 1966.

92- Coval, M. L.;Taurog, A. Purification and iodinating activity of hog thyroid peroxidase. J Biol Chem., v.242, p.5510, 1967. 
93-DeGroot, L. J.; Davis, A.M. Studies on the biosynthesis of iodotyrosines: A soluble thyroid iodideperoxidase tyrosine-iodinase system. Endocrinology., v.70, p.492, 1962.

94-Mahoney, C. P.; Igo, R.P. Studies of thebiosynthesis of thyroxine. II. Solubilization and characterization of an iodide peroxidase from thyroid tissue, Biochim Biophys Ada., v.113, p.507, 1966.

95- Berry, M.N.; Gregory, R.B. On the thyroid hormone-induced increase in respiratory capacity of isolated rat hepatocytes. Biochim Biophys Acta., v.1098, p.61-7,1991.

96- Panveloski-Costa, A.C.; Serrano-Nascimento, C.; Bargi-Souza, P.; Poyares, L.L.;Viana, G.S.; Nunes, M.T. Beneficial effects of thyroid hormone on adipose inflammation and insulin sensitivity of obese Wistar rats. Physiol Rep., v.6, 2018.

97- Prieto-Almeida, F.; Panveloski-Costa, A.C.; Crunfli, F.; da Silva Teixeira, S.; Nunes, M.T.; Torrão, A.S. Thyroid hormone improves insulin signaling and reduces the activation of neurodegenerative pathway in the hippocampus of diabetic adult male rats. Life Sci., v.192, p.253-258, 2018.

98- Peliciari-Garcia, R.A.; Bargi-Souza, P.; Young, M.E.; Nunes, M.T. Repercussions of hypo and hyperthyroidism on the heart circadian clock. Chronobiol ., v.35, p.147-159, 2018.

99- Funes-Huacca, M.; Regitano, L.C.A.; Mueller, O.; Carrilho, E. Semiquantitative determination os Alicylobacillus acidoterrestris in orange juice by reverse transcriptase polymerase chain reaction and capillary electrophoresis - laser induced fluorescence using microchip technology. Electrophoresis., v. 25, p.3860-3864, 2004.

100- Kambach, C.; Walke, S.; Young, R.; Avis, J.M.; delaFortelle, E.; Raker, V.A.; Luhrmann, R.; Li, J.; Nagai, K. Crystal structures of two Sm protein complexes and their implications for the assembly of the spliceosomal snRNPs, Cell., v.96, p.375-387, 1999.

99-Bianco, A.C.; Salvatore, D.; Gereben, B.; Berry, M.J.; Larsen, P.R. Biochemistry, cellular and molecular biology, and physiological roles of the iodothyronine selenodeiodinases. Endocr Rev., v.23, p.38-89, 2002.

100-Zavacki, A.M.; Ying, H.; Christoffolete, M.A.; Aerts, G.; So, E.; Harney, J.W.; Cheng, S.Y.; Larsen, P.R.; Bianco, A.C. Type 1 iodothyronine deiodinase is a sensitive marker of peripheral thyroid status in the mouse. Endocrinology., v.146, p.1568-75, 2005. 
101-Weissman, A.M. Themes and variations on ubiquitylation. Nat Rev Mol Cell Biol., v.2, p.169-78, 2001.

102-Huibregtse, J. M.; Scheffner, M.; Beaudenon, S.; Howley,P. M. A family of proteins structurally and functionally related to the E6-AP ubiquitin-protein ligase. Proc. Natl Acad. Sci. USA., v.92, p.2563-2567,1995.

103-Joazeiro, C. A.; Weissman, A. M. RING finger proteins: mediators of ubiquitin ligase activity. Cell., v.102, p.549-552, 2000.

104-Bienz, M. The PHD finger, a nuclear protein-interaction domain. TRENDS in Biochemical Sciences., v..31, p. 35-40, 2006.

105- Carrascosa, J.L.; Llorca, O.; Valpuesta, J.M. Structural comparison of prokaryotic and eukaryotic chaperonins. Micron., v.32, p.43-50, 2001.

106- Valpuesta, J.M.; Martín-Benito, J.; Gómez-Puertas, P.; Carrascosa, J.L.; Willison, K.R. Structure and function of a protein folding machine: the eukaryotic cytosolic chaperonin CCT. FEBS Lett., v.529, p.11-6, 2002.

107- Bukau, B.; Horwich, A.L. The Hsp70 and Hsp60 chaperone machines. Cell., v.92, p.351-66, 1998.

108- Braig, K.; Otwinowski, Z.; Hegde, R.; Boisvert, D.C.; Joachimiak, A.; Horwich, A.L.; Sigler, P.B. A mutant at position 87 of the GroEL chaperonin is affected in protein binding and ATP hydrolysis. Nature., v.371, p.578-586, 1995.

109-Xu, Z.; , Horwich, A.L.; Sigler, P.B. The crystal structure of the asymmetric GroEL-GroES-(ADP)7 chaperonin complex. Nature., v.388, p.741-50, 1997.

110- Sigler, P.B.; Xu, Z.; Rye, H.S.; Burston, S.G.; Fenton, W.A.; Horwich, A.L. Structure and function in GroEL-mediated protein folding. Annu Rev Biochem., v.67, p.581-608, 1998.

111- Grallert, H.; Buchner, J. Review: a structural view of the GroE chaperone cycle. J Struct Biol., v.135, p.95-103, 2001.

112-Willison, K.R. and Horwich, A.L. The Chaperonins., Academic Press, San Diego, pp. 107-136, 1996.

113- Gutsche, I.; Essen, L.O.; Baumeister, W. Group II chaperonins: new TRiC(k)s and turns of a protein folding machine. J Mol Biol., v.293, p.295-312, 1999.

114- Klumpp, M.; Baumeister, W.; Essen, L.O. Structure of the substrate binding domain of the thermosome, an archaeal group II chaperonin. Cell., v.91, p.263-70, 1997. 
115- Ditzel, L.; Löwe, J.; Stock, D.; Stetter, K.O.; Huber, H.; Huber, R.;

Steinbacher, S. Crystal structure of the thermosome, the archaeal chaperonin and homolog of CCT. Cell., v.93, p.125-38, 1998.

116-Vainberg, I. E.; Lewis, S. A.; Rommelaere, H.; Ampe, C.; Vandekerckhove, J.; Klein, H. L.; Cowan, N. J. Prefoldin, a chaperone that delivers unfolded proteins to cytosolic chaperonin. Cell., v.93, p.863-873, 1998.

117-Gao, Y.; Thomas, J. O.; Chow, R. L.; Lee, G. H.; Cowan, N. J. A cytoplasmic chaperonin that catalyzes beta-actin folding. Cell., v.69, p.10431050, 1998.

118-Chen, X.; Sullivan, D. S.; Huffaker, T. C. Two yeast genes with similarity to TCP-1 are required for microtubule and actin function in vivo. Proc. Natl. Acad. Sci. U.S.A., v.91, p. 9111-9115, 1994.

119-Geissler, S.; Siegers, K.; Schiebel, E. A novel protein complex promoting formation of functional alpha- and gamma-tubulin. EMBO. J., v.17, p.952-966, 1998.

120-Siegers, K.; Waldmann, T.; Leroux, M. R.; Grein, K.; Shevchenko, A.; Schiebel, E.; Hartl, F. U. Compartmentation of protein folding in vivo: sequestration of non-native polypeptide by the chaperonin-GimC system. EMBO. J.,v.18, p.75-84,1999.

121-Siegert, R.; Leroux, M. R.; Scheufler, C.; Hartl, F. U.; Moarefi, I. Structure of the molecular chaperone prefoldin: unique interaction of multiple coiled coil tentacles with unfolded proteins. Cell., v.103, p.621-632, 2000.

122-Martín-Benito, J.; Boskovic, J.; Go'mez-Puertas, P.; Carrascosa, J. L.; Simons, C. T.; Lewis, S. A.; Bartolini, F.; Cowan, N. J.; Valpuesta, J. M. Structure of eukaryotic prefoldin and of its complexes with unfolded actin and the cytosolic chaperonin CCT. EMBO. J., v.21, p.6377-6386, 2002.

123-Leroux, M. R.; Fa“ndrich, M.; Klunker, D.; Siegers, K.; Lupas, A. N.; Brown, J. R.; Schiebel, E.; Dobson, C, M.; Hartl, F. U. MtGimC, a novel archaeal chaperone related to the eukaryotic chaperonin cofactor GimC/prefoldin.

EMBO. J., v.18, p.6730-6743, 1999.

124- Cao, J.; Xu, J.; Li, W.; Liu, J. Influence of selective brain cooling on the expression of ICAM-1 mRNA and infiltration of PMNLs and monocytes/macrophages in rats suffering from global brain ischemia/reperfusion injury. Biosci Trends., v.2, p.241-4, 2008.

125- Furuichi, Y.; Shatkin, A. J. Viral and cellular mRNA capping: past and prospects. Adv. Virus Res. v.55, p.135-184, 2000.

126-Shatkin, A. J. Capping of eucaryotic mRNAs. Cell., v.9, p.645-653,1976. 
127-Shibagaki, Y.; Itoh, N.; Yamada, H.; Nagata, S.; Mizumoto, K. mRNA capping enzyme. Isolation and characterization of the gene encoding mRNA guanylytransferase subunit from Saccharomyces cerevisiae. J. Biol. Chem., v.267, p.9521-9528, 1992.

128-Tsukamoto, T.; Shibagaki, Y.; Imajoh-Ohmi, S.; Murakoshi, T.; Suzuki, M.; Nakamura, A.; Gotoh, H.; Mizumoto, K. Isolation and characterization of the yeast mRNA capping enzyme beta subunit gene encoding RNA 5triphosphatase, which is essential for cell viability. Biochem. Biophys. Res. Commun., v.239, p.116-122, 1997.

129-Mao, X.; Schwer, B.; Shuman, S. Yeast mRNA cap methyltransferase is a 50 kilodalton protein encoded by an essential gene. Mol. Cell. Biol., v.15, p.4167-4174,1995.

130-Konarska, M. M.; Padgett, R. A.; Sharp, P. A. Recognition of cap structure in splicing in vitro of mRNA precursors. Cell., v.38, p.731-736,1984.

131-Edery, I.; Sonenberg, N. Cap-dependent RNA splicing in a HeLa nuclear extract. Proc. Natl. Acad. Sci. U.S.A., v.82, p.7590-7594,1985.

132-Ohno, M.; Sakamoto, H.; Shimura, Y. Preferential excision of the 5proximal intron from mRNA precursors with two introns as mediated by the cap structure. Proc. Natl. Acad. Sci. U.S.A., v.84, p.5187-5191,1987.

133-Spriggs, K. A.; Stoneley, M.; Bushell, M.; Willis, A. E. Re-programming of translation following cell stress allows IRES-mediated translation to predominate. Biol. Cell., v.100, p.27-38, 2008.

134-Muthukrishnan, S.; Both, G. W.; Furuichi, Y.; Shatkin, A.J. 5-Terminal 7methylguanosine in eukaryotic mRNA is required for translation. Nature., v.255, p.33-37, 1975.

135-Both, G. W.; Banerjee, A. K.; Shatkin, A. J. Methylation-dependent translation of viral messenger RNAs in vitro. Proc. Natl. Acad. Sci. U.S.A., v.72, p.1189-1193,1975.

136-Gonatopoulos-Pournatzis,T.;Dunnn, S.; Bounds, R.; Cowling,V.H. RAM/Fam103a1 Is Required for mRNA Cap Methylation. Molecular Cell., v.44, p.585-596, 2011.

137-Di Cosmo, C.; Liao, X.H.; Dumitrescu, A.M.; Philp, N.J.,;Weiss, R.E.; Refetoff, $\mathrm{S}$. Mice deficient in MCT 8 reveal a mechanism regulating thyroid hormone secretion. J Clin Invest., v.120, p.3377-3388, 2010.

138-Nishimura, M.; Naito, S. Tissue-specific mRNA expression profiles of human solute carrier transporter superfamilies. Drug Metab Pharmacokinet.,v. 23, p.22-44, 2008. 
139-Bianco, A.C.; Salvatore, D.; Gereben, B.; Berry, M.J.; Larsen, P.R. Biochemistry, cellular and molecular biology, and physiological roles of the iodothyronine selenodeiodinases. Endocr Rev., v.23, p.38-89, 2002.

140-Zavacki, A.M.; Ying, H.; Christoffolete, M.A.; Aerts, G.; So, E.; Harney, J.W.; Cheng, S.Y.; Larsen, P.R.; Bianco, A.C. Type 1 iodothyronine deiodinase is a sensitive marker of peripheral thyroid status in the mouse. Endocrinology., v. 146, p.1568-75, 2005

141- Hu, Z.; Zhuo, X.; Shi, Y.; Liu, X.; Yuan, J.; Li, L.; Sun, Y. Iodine deficiency up-regulates monocarboxylate transporter 8 expression of mouse thyroid gland. Chin Med J., v.127, p.4071-6. 2014.

142- Shiraishi, M.; Yamamoto, Y.; Hirooka, N.; Obuchi, Y.; Tachibana, S.; Makishima, M.; Tanaka, Y. A high concentration of triiodothyronine attenuates the stimulatory effect on hemin-induced erythroid differentiation of human erythroleukemia K562 cells. Endocr J., v.62, p.431-40, 2015.

143- Panveloski-Costa, A.C.; Silva Teixeira, S.; Ribeiro, I.M.; SerranoNascimento, C.; das Neves, R.X.; Favaro, R.R.; Seelaender, M.; Antunes, V.R.; Nunes, M.T. Thyroid hormone reduces inflammatory cytokines improving glycaemia control in alloxan-induced diabetic wistar rats. Acta Physiol., v.217, p.130-40, 2016.

144- Visser, W.E.; Friesema, E.C.; Jansen, J.; Visser, T.J. Thyroid hormone transport by monocarboxylate transporters. Best Pract Res Clin Endocrinol Metab., v.21, p.223-36, 2007.

145- Braun, D.; Kim, T.D.; le Coutre, P.; Köhrle, J.; Hershman, J.M.; Schweizer, $U$. Tyrosine kinase inhibitors noncompetitively inhibit MCT 8-mediated iodothyronine transport. J Clin Endocrinol Metab., v.97, p.2011-1837, 2012.

146-McDonough, A.A. T. A. Brown B. Horowitz R. Chiu J. Schlotterbeck J. Bowen C. A. Schmitt. Thyroid hormone coordinately regulates $\mathrm{Na+-}+-$ ATPase alpha- and beta-subunit mRNA levels in kidney. Am J Physiol.,v.254, p.323-329, 1988.

147-Will, C.L.; Behrens, S.E.; Lu"hrmann, R. Protein composition of mammalian spliceosomal snRNPs. Mol. Biol. Rep., v.18, p.121-126,1993.

148-Lerner, M.R.; Steitz, J.A. Antibodies to small nuclearRNAs complexed with proteins are produced by patients with systemic lupus erythematosus. Proc. Natl. Acad. Sci. USA., v.76, p.5495-5499,1979.

149-Craft, J. Antibodies to snRNPs in systemic lupus erythemato-sus. Rheum. Dis. Clin. North Am., v.18, p.311-335,1992. 
150-Zieve, G.W.; Sauterer, R.A. Cell biology of the snRNP particles. CRC Crit. Rev. Biochem. Mol.Biol., v.25, p.1-46,1990.

151-Krämer, A. The structure and function of proteins involved in mammalian pre-mRNA splicing. Annu. Rev. Biochem., v.65, p.367-409,1996.

152-Burge, C.B.; Tuschl, T.; Sharp, P.A.. Splicing of precursors to mRNAs by the spliceosomes., pp. 525-560,1999.

153-Patel, A.A.; Steitz, J.A. Splicing double:Insights from the second spliceosome. Nat. Rev. Mol. Cell Biol., v.4, p.960-970, 2003.

154-Gozani, O.; Feld, R.; Reed, R. Evidence that sequence-independent binding of highly conserved U2 snRNP proteins upstream of the branch site is required for assembly of spliceosomal complex A. Genes Dev., v.10, p.233243, 1996.

155- Valcarcel, J.; Gaur, R.K.; Singh, R.; Green, M.R. Interaction of U2AF65 RS region with pre-mRNA branch point and promotion of base pairing with $\mathrm{U} 2$ snRNA. Science., v.273, p.1706-1709,1996.

156- Gozani, O.; Potashkin, J.; Reed, R. A potential role for U2AF-SAP 155 interactions in recruiting U2 snRNP to the branch site. Mol. Cell. Biol., v.18, p. 4752-4760,1998.

157-Umen, J.G.; Guthrie, C. The second catalytic step of pre-mRNA splicing. RNA., v.1, p.869-885,1995.

158- Rokeach, L.A.; Haselby, J.A.; Hoch, S.O. Molecular cloning of a cDNA encoding the human Sm-D autoantigen. Proc Natl Acad Sci U S A., v.85, p. 4832-6, 1988.

159-Lehmeier, T.; Kirani Foulaki and Reinhard LGhrmann Evidence for three distinct $D$ proteins, which react differentially with anti-Sm autoantibodies, in the cores of the major snRNPs UI, U2, U4/U6 and U5. Nucleic Acids Research., v.18,p.6475-6484, 1990.

160-Vanin, E.F. Processed pseudogenes: characteristics and evolution. Annu Rev Genet., v.19, p.253-72,1985.

161-Kim, Y.D.; Lee, J.; Kim, H.S.; Lee, M.O.; Son, M.Y.; Yoo, C.H.; Choi, J.K.; Lee, S.C.; Cho, Y.S. The unique spliceosome signature of human pluripotent stem cells is mediated by SNRPA1, SNRPD1, and PNN. Stem Cell Res., v.22, p.43-53, 2017.

162-Quidville, V.; Alsafadi, S.; Goubar, A.; Commo, F.; Scott1, V.; PiocheDurieu, C.; Girault, I.; Baconnais, S.; Cam, E.L.; Lazar, V.; Delaloge, S.; Saghatchian, M.; Pautier, P.; Morice, P.; Dessen, P.; Vagner, S.; Andre, F. 
Targeting the Deregulated Spliceosome Core Machinery in Cancer Cells Triggers mTOR Blockade and Autophagy. Cancer research., v.73, p.22472258, 2013.

163- Hör, S.; Ziv, T.; Admon, A.; Lehner, P.J. Stable isotope labeling by amino acids in cell culture and differential plasma membrane proteome quantitation identify new substrates for the MARCH9 transmembrane E3 ligase. Mol Cell Proteomics., v.8, p.1959-71, 2009.

164-Thien, C.B.; Langdon, W.Y. c-Cbl and Cbl-b ubiquitin ligases: substrate diversity and the negative regulation of signalling responses. Biochem. J., v.391,p.153-166, 2005.

165-Joazeiro, C.A.; Wing, S.S.; Huang, H.; Leverson, J.D.; Hunter, T.; Liu, Y.C. The tyrosine kinase negative regulator c-Cbl as a RINGtype, E2-dependent ubiquitin-protein ligase. Science., v.286, p.309-312, 1999.

166-Levkowitz, G.; Waterman, H.; Ettenberg, S.A.; Katz, M.; Tsygankov, A.Y.; et al. Ubiquitin ligase activity and tyrosine phosphorylation underlie suppression of growth factor signaling by c-Cbl/Sli-1. Mol. Cell., v.4,p.1029-1040, 1999.

167-Rao, N.; Ghosh, A.K.; Ota, S.; Zhou, P.; Reddi, A.L.; et al. The nonreceptor tyrosine kinase Syk is a target of Cbl-mediated ubiquitylation upon Bcell receptor stimulation. EMBO J., v.20, p.7085-7095, 2001.

168-Nathan, J.A.; Lehner, P.J. The trafficking and regulation of membrane receptors by the RING-CH ubiquitin E3 ligases. Exp Cell Res., v.315, p.15931600, 2009.

169-Chang, H.; Dittmer, D.P.; Shin, Y.C.; Chul, S.Y.; Hong, Y. Role of Notch signal transduction in Kaposi's sarcoma-associated herpesvirus gene expression. J Virol., v.79, p.14371-14382, 2005.

170-Chang, H. Notch signal transduction induces a novel profile of Kaposi's sarcoma-associated herpesvirus gene expression. J Microbiol., v.44, p.217225, 2006. 